\title{
A Novel Variable Index and Excision CFAR Based Ship Detection Method on SAR Imagery
}

\author{
Kefeng Ji, Xiangwei Xing, Huanxin Zou, and Jixiang Sun \\ College of Electronic Science and Engineering, National University of Defense Technology, Changsha, Hunan 410073, China \\ Correspondence should be addressed to Kefeng Ji; jikefeng@nudt.edu.cn
}

Received 10 December 2014; Revised 3 March 2015; Accepted 3 March 2015

Academic Editor: Ignacio R. Matias

Copyright ( 2015 Kefeng Ji et al. This is an open access article distributed under the Creative Commons Attribution License, which permits unrestricted use, distribution, and reproduction in any medium, provided the original work is properly cited.

\begin{abstract}
When applying the constant false alarm rate (CFAR) detector to ship detection on synthetic aperture radar (SAR) imagery, multiple interferers such as upwelling, breaking waves, ambiguities, and neighboring ships in a dense traffic area will degrade the probability of detection. In this paper, we propose a novel variable index and excision CFAR (VIE-CFAR) based ship detection method to alleviate the masking effect of multiple interferers. Firstly, we improve the variable index (VI) CFAR with an excision procedure, which censors the multiple interferers from the reference cells. And then, the paper integrates the novel CFAR concept into a ship detection scheme on SAR imagery, which adopts the VIE-CFAR to screen reference cells and the distribution to derive detection threshold. Finally, we analyze the performances of the VIE-CFAR under different environments and validate the proposed method on both ENVISAT and TerraSAR-X SAR data. The results demonstrate that the proposed method outperforms other existing detectors, especially in the presence of multiple interferers.
\end{abstract}

\section{Introduction}

Ship detection filters the peaks among sea clutter to indicate the location of potential ships. With the development of remote sensing technology, ship detection on remote sensing imagery becomes more and more attractive. Among others, synthetic aperture radar (SAR) sensors are particularly appealing due to the fact that they are independent of atmospheric and meteorological conditions $[1,2]$. Therefore, ship detection on SAR imagery has received a lot of interests in the past decades [3-6]. To the present date, the European Commission and European Space Agency have jointly initiated series of projects [7], such as IMPAST, DECLIMS, and LIMES, to develop efficient and reliable maritime surveillance systems, in which ship detection on SAR imagery is one of the most critical modules.

Besides the methods based on wavelet analysis [8] and subaperture correlation [9], constant false alarm rate (CFAR) method is the most popular technique for ship detection on SAR imagery [3]. The CFAR scheme sets the detection threshold adaptively based on the local statistic, which is estimated by processing a group of reference cells surrounding the cell under test (CUT). In a homogeneous environment when the reference cells contain independent and identically distributed samples governed by an exponential distribution, it has been shown in [10] that the cell averaging (CA) CFAR can achieve the near optimum performance. However, the CA-CFAR detector will be deteriorated under nonhomogeneous environments, for example, clutter edges and multiple interferers [11-13]. The nonhomogeneous environments result in either an excessive increase in false alarms or degradation of detection probability. To overcome these problems, alternative CFAR processors are proposed. The great-of (GO), smallest-of (SO), order statistic (OS), and trimmed mean (TM) CFARs are the most representative ones. These CFAR detectors produce diverse performances under different clutter environments [11]. To exploit the advantages of different CFAR detectors for accommodating the variety of environments, an intelligent CFAR concept based on data variability index (VI) and mean ratio (MR) of the leading and lagging windows, named VI-CFAR, is proposed in [14], which assesses the current environment and switches among the CA-CFAR, GO-CFAR, and SOCFAR dynamically. The VI-CFAR provides low CFAR loss in homogeneous environments and performs robustly in the presence of clutter edges and properly located multiple 


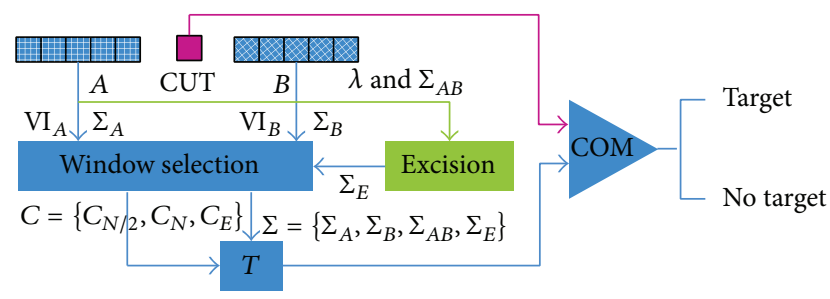

FIGURE 1: Illustration of the VIE-CFAR scheme.

interferers. However, if the interferers are located in both sides of the reference window, the performance of VI-CFAR degrades with the increasing number of interferers.

Practical ship detection on SAR imagery often encounters interferers such as upwelling, breaking waves, ambiguities, and neighboring ships in a dense traffic area. On these occasions, when applying the CFAR scheme to ship detection on SAR imagery, the interferers are likely to produce a higher detection threshold, corresponding to the masking effect in the detection issue. To overcome the aforementioned problems, we propose a novel variability index and excision CFAR (VIE-CFAR) based ship detection method on SAR imagery. The proposed method firstly incorporates the VI-CFAR with an excision procedure to filter the reference cells. And then, we derive the detection threshold based on the survived reference cells and $G^{0}$ distribution [15]. The proposed method addresses the ship detection on SAR imagery in the presence of multiple interferers and inherits the robust performance of VI-CFAR in various environments. We analyze the proposed method under different environments and validate its performances on both ENVISAT and TerraSAR-X SAR data.

The rest of this paper is organized as follows. Section 2 presents our proposed VIE-CFAR scheme in detail. In Section 3, we integrate the VIE-CFAR into a ship detection flowchart. In Section 4, simulation experiments are performed in various environments. Section 5 conducts the validation of the VIE-CFAR for ship detection on ENVISAT and TerraSAR-X SAR imagery. Finally, Section 6 concludes this paper.

\section{Description of the VIE-CFAR Scheme}

In this paper, we present the VIE-CFAR scheme in Figure 1, which incorporates the VI-CFAR with an excision procedure. Throughout this paper, we use the similar notations as in [14]. In the VIE-CFAR scheme, we check whether a target exists for each CUT by comparing the CUT $Y$ with a threshold $T$. If $Y>T$, then a target can be declared to be present. Here, the threshold $T$ is decided by the estimated statistic of the reference cells $\left\{X_{i} \mid i=1,2, \ldots, N\right\}$ and a multiplier constant $C$. In most cases, the statistic is estimated as the sum of the reference cells. Furthermore, in the proposed VIECFAR scheme, the reference window can be selected as the whole window $A B$, the leading window $A$, the lagging window $B$, or the excision window $E$ (results from the whole window $A B$ and an excision parameter $\lambda$ ) based on the data VI and $\mathrm{MR}$ of the leading and lagging windows. Correspondingly, the CFAR processor switches among CA-CFAR, GO-CFAR, SO-CFAR, and excision-CFAR (E-CFAR) dynamically.

2.1. Definition of VI and MR. We adopt the definition of the VI and MR detailed in [14]. VI is a second-order statistic and its value is a function of the estimated population mean $\widehat{u}$ and variance $\widehat{\sigma}^{2}$. Specifically, the VIE-CFAR calculates the VI of the leading and lagging window, respectively, as

$$
\mathrm{VI}=1+\frac{\widehat{\sigma}^{2}}{\widehat{u}^{2}}=1+\frac{1}{n-1} \sum_{i=1}^{n} \frac{\left(X_{i}-\bar{X}\right)^{2}}{(\bar{X})^{2}},
$$

where $\bar{X}$ is the arithmetic mean of the $n=N / 2$ cells in a half reference window.

In a homogeneous environment, the probability density function (PDF) of VI is independent of the noise power. However, the value of VI changes considerably when clutter edges and interferers are present. Therefore, we need to decide if the environment of the half reference window is variable or nonvariable. This is done by comparing VI with a threshold $K_{\mathrm{VI}}$ via applying the following hypothesis [14]:

$$
\begin{gathered}
\mathrm{VI} \leq K_{\mathrm{VI}} \Longrightarrow \text { Non-variable, } \\
\mathrm{VI} \geq K_{\mathrm{VI}} \Longrightarrow \text { Variable. }
\end{gathered}
$$

The MR is further defined to determine whether the cells in the leading and lagging windows come from the same environment or not. It is defined as the ratio of the mean values of the leading and the lagging window cells, denoted by $[14]$ :

$$
\mathrm{MR}=\frac{\bar{X}_{A}}{\bar{X}_{B}}=\frac{\sum_{i \in A} X_{i}}{\sum_{i \in B} X_{i}}
$$

in which $\bar{X}_{A}$ and $\bar{X}_{B}$ are the leading and lagging window means. Similar to VI, the PDF of MR is independent of the noise power in a homogeneous environment. However, the value of MR increases when interfering targets and higher power of clutters are present in the leading window while it decreases when they are present in the lagging window.

To determine whether the means of the leading and lagging windows are the same or not, we use the following hypothesis test by comparing the MR with a threshold $K_{\mathrm{MR}}$ and its reciprocal [14]:

$$
\begin{gathered}
K_{\mathrm{MR}}^{-1} \leq \mathrm{MR} \leq K_{\mathrm{MR}} \Longrightarrow \text { Same Means, } \\
\mathrm{MR}<K_{\mathrm{MR}}^{-1} \text { or } \mathrm{MR}>K_{\mathrm{MR}} \Longrightarrow \text { Different Means. }
\end{gathered}
$$

2.2. Window Selection Strategy and Excision Procedure. In this section, we present the window selection strategy of the VIE-CFAR scheme. In contrast to the VI-CFAR that selects different reference windows to estimate the background statistic based on the VI and MR [14], we improve it by introducing an excision procedure, as shown in Table 1. In the case of multiple interfering targets, especially when the interferers are present in both the leading and lagging 
TABLE 1: Multiplier and window selection.

\begin{tabular}{lcccc}
\hline $\begin{array}{l}\text { Leading window } \\
\text { variable }\end{array}$ & $\begin{array}{c}\text { Lagging window } \\
\text { variable }\end{array}$ & Different means & $\begin{array}{c}\text { VIE-CFAR adaptive } \\
\text { thresholds }\end{array}$ & $\begin{array}{c}\text { Equivalent CFAR } \\
\text { detector }\end{array}$ \\
\hline No & No & No & $C_{N} \Sigma_{A B}$ & CA-CFAR \\
No & No & Yes & $C_{N / 2} \max \left(\Sigma_{A}, \Sigma_{B}\right)$ & GO-CFAR \\
Yes & No & - & $C_{N / 2} \Sigma_{B}$ & CA-CFAR \\
No & Yes & - & $C_{N / 2} \Sigma_{A}$ & CA-CFAR \\
Yes & Yes & - & $C_{E} \Sigma_{E}$ & E-CFAR \\
\hline
\end{tabular}

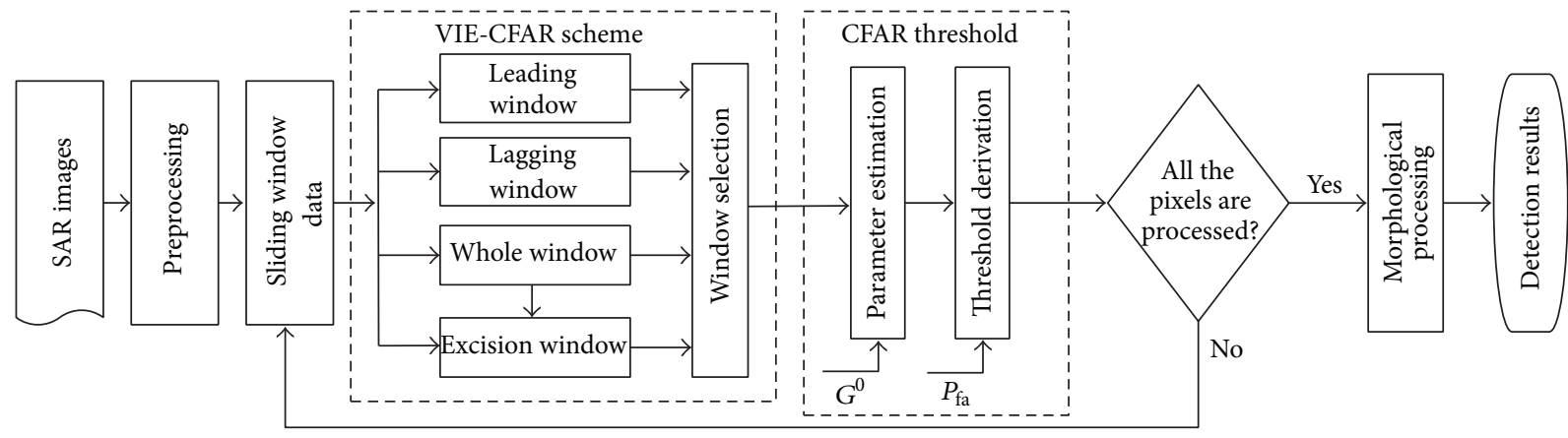

FIGURE 2: Block diagram of the proposed method.

windows, the VI-CFAR chooses the half window with the smallest mean and thus results in a loss in detection probability. To avoid this drawback, we propose to use an excision procedure [16] for suppressing the masking effect caused by the interfering targets. The introduced excision procedure is referred to as E-CFAR. This method compares the samples in the whole window $A B$ with an excision threshold and groups the samples that do not exceed the excision threshold into an excision window denoted by $E$ with the size of $N_{E}$. We can explicitly define the excision window as

$$
E=\left\{X_{i} \mid X_{i} \leq \lambda \sum_{i \in A B} X_{i}\right\},
$$

where $\lambda$ is the excision coefficient.

Here the value of $\lambda$ is set based on the sum of the reference cells and an excision probability to ensure the survived samples in window $E$ are not variable. More specifically, we select the value of $\lambda$ based on the following steps.

Firstly, we set a relative low excision probability, for example, $1 e-6$, and accordingly calculate an initial excision coefficient $\lambda^{(0)}$ (similar to (7) below):

$$
\lambda^{(i)}=\left(P_{\mathrm{fa}}\right)^{-1 / N_{E}^{(i)}}-1, \quad i=0,1,2, \ldots, M
$$

in which $i$ denotes the $i$ th iteration and $N_{E}^{(i)}$ denotes the number of the survived cells of the $i$ th iteration and $N_{E}^{(0)}=N$.

Secondly, we screen the reference cells based on (5) to obtain the excision window $E$ and calculate VI of the survived reference cells.

Thirdly, we compare the value of VI with $K_{\mathrm{VI}}$ to determine the variability of $E$. If the window $E$ is variable, we increase the excision probability with a step of 5 times of the initial value (e.g., if the initial value is $1 e-6$, the step is $5 e-6$ ) and recalculate a lower $\lambda$.

And the second and third steps repeat until the reference cells are not variable. With the iteratively calculated $\lambda$, the interferers can be removed and accordingly the survived reference cells are in nonvariable environment.

Besides the selected subset of reference cells to estimate the background statistic, the VIE-CFAR also determines a multiplier constant to form the adaptive detection threshold. Under the assumption that the background cells are governed by an exponential distribution, we determine the multiplier constant $C_{N}$ based on CA-CFAR with reference cells [14]:

$$
C_{N}=\left(P_{\mathrm{fa}}\right)^{-1 / N}-1,
$$

where $P_{\mathrm{fa}}$ is the predetermined probability of false alarm (PFA). It is worth noting that the number of reference cells in window $E$ is $N_{E}$ and the multiplier $C_{E}$ is calculated from (6) by replacing $N$ with $N_{E}$.

\section{VIE-CFAR for Ship Detection in SAR Images}

Based on the VIE-CFAR scheme described above, we propose a VIE-CFAR based ship detection method on SAR imagery. Figure 2 illustrates the block diagram of the proposed method. When applying the VIE-CFAR to ship detection on SAR imagery, we further improve the procedures in the following aspects. Firstly, we extend the 1D reference window to a $2 \mathrm{D}$ hollow stencil and obtain the reference cells based on the proposed VIE-CFAR scheme. Secondly, we adopt the $G^{0}$ distribution to describe the statistical characteristic of sea clutter. Thirdly, the method estimates the parameters of $G^{0}$ distribution and calculates the decision threshold to filter ships from sea clutter. Finally, the CFAR detection results are 


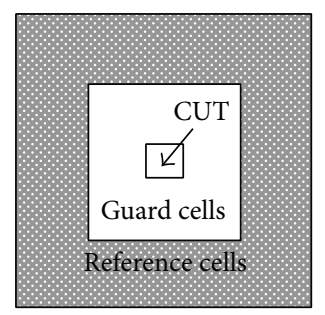

(a)

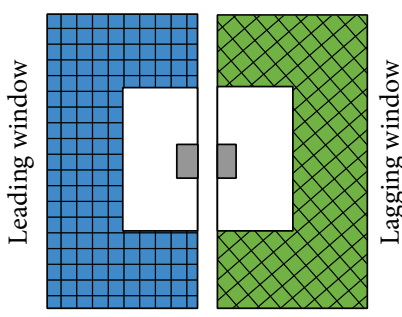

(b)

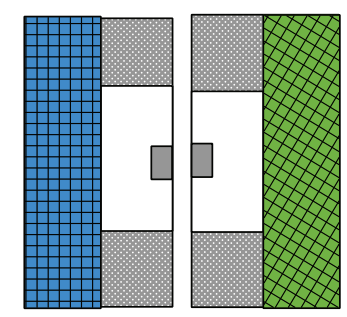

(c)

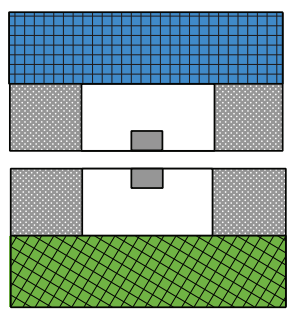

(d)

Figure 3: Illustration of the 2D stencil: (a) the three-layer sliding window, (b) leading and lagging window, and (c) and (d) variations of the sliding window.

postprocessed with morphological operations to cluster and filter isolated pixels.

As the SAR sensor captures the observed area from both range and azimuth directions, the VIE-CFAR reference window for ship detection on SAR image is extended to a two-dimensional hollow stencil, as shown in Figure 3(a). The stencil consists of three layers [3], where the central one concentrates on the pixels under test, and the guard window protects the target from leaking to the background window. The stencil size depends on the image resolution and ship target dimensions. The leading and lagging windows are illustrated in Figure 3(b). Notice that the leading and lagging window can be defined as other forms to be more robust to clutter edges, as shown in Figures 3(c) and 3(d). Due to the fact that the wave fields present various directions on SAR images (not only limited to horizontal or vertical), the stencil window should be designed flexibly to remain consistent with the wave field direction.

The adopted statistical model is another important factor that impacts ship detection performance on SAR imagery. Conducted from the multiplicative model and the underlying physical scattering mechanisms, the $G^{0}$ distribution has arisen to represent clutters on SAR imagery [15]. The PDF of a random variable $X$ that has an amplitude $G^{0}$ distribution is denoted as

$$
f_{X}(x)=\frac{2 L^{L} \Gamma(L-\alpha) x^{2 L-1}}{\gamma^{\alpha} \Gamma(L) \Gamma(-\alpha)\left(\gamma+L x^{2}\right)^{L-\alpha}}, \quad-\alpha, \gamma, L, x>0,
$$

where $L$ denotes the equivalent number of looks (ENL) of the amplitude image, $\alpha$ is a shape parameter that describes the spikiness of the clutter, $\gamma$ is a scale parameter, and $\Gamma(\cdot)$ is the gamma function. The $G^{0}$ distribution is compatible with several special cases when the shape parameter adopts different values. The exponential distribution is one of the special cases of $G^{0}$ distribution [17]. The parameters are estimated from sea clutters by Mellin kind statistics [18].

Different from the one-dimensional CFAR detection, the $2 \mathrm{D}$ stencil ensures enough reference cells for the estimation of the background statistics. Therefore, we estimate the shape and scale parameters with different reference cells based on the switch logic in Table 1 . And then we directly derive the detection threshold $T_{d}$ for ship detection on SAR imagery by (8):

$$
P_{\mathrm{fa}}=\int_{T_{d}}^{\infty} f_{X}(x) d x=\int_{T_{d}}^{\infty} \frac{2 L^{L} \Gamma(L-\alpha) x^{2 L-1}}{\gamma^{\alpha} \Gamma(L) \Gamma(-\alpha)\left(\gamma+L x^{2}\right)^{L-\alpha}} d x
$$

When $L=1$, close form of (8) can be derived

$$
P_{\mathrm{fa}}=\frac{\alpha-1}{\alpha \gamma^{\alpha}}\left(\gamma+T_{d}^{2}\right)^{\alpha} ;
$$

therefore, $T_{d}$ is presented as

$$
T_{d}=\left(\frac{\alpha \gamma^{\alpha}}{\alpha-1} P_{\mathrm{fa}}^{1 / \alpha}-\gamma\right)^{1 / 2} .
$$

However, analytical expression of (8) is difficult to be solved for most cases. Once given the PFA, we derive the decision threshold $T_{d}$ based on (8) with numerical calculations. Ship target is declared to be present if the image pixel value exceeds the decision threshold.

\section{Simulation Results and Analysis}

In this section, we illustrate the performances of VIE-CFAR for the environments of homogeneous, clutter edges and multiple interferers. We compare the probability of detection (PD) and the PFA performances of the VIE-CFAR with the existing CFAR detectors, including the CA, GO, SO, OS $(k=$ $21, k$ is the order of the cell being taken as the estimation of local statistic), and VI-CFAR.

Due to the lack of analytical expression for the PDF of VI and the dependence between the VI and MR hypothesis test decisions [14], we apply the Monte-Carlo simulation $(M=1,000,000)$. In these simulations, we mainly expect to demonstrate the ability of selecting homogeneous clutter cells by the VIE-CFAR scheme. Therefore, we generally assume the exponential distribution instead of the $G^{0}$ distribution for background cells and Swerling I fluctuation for the target and interferers. The interferer to noise ratio (INR) equals the signal to noise ratio (SNR). The interferers are generated randomly and inserted to the appointed cells. The clutter edge sweeps from left to right (Window $A$ to Window $B$ ) with clutter to noise ratio (CNR) as $10 \mathrm{~dB}$. The multiplier constants are calculated from (6) given that the number of 
reference cells is $N=24$ and the designed $P_{\mathrm{fa}}$ is $1 e-4$. As described in [14], the values of VI and MR are related to the CNR and INR of the variable environments, as well as the number of reference cells. We here set them as 4.76 and 1.806, respectively. The excision coefficient $\lambda$ is calculated through the iteration procedure in Section 2.2 with an initial excision probability of $1 e-6$.

Figure 4 illustrates the various environments of the simulation, including the homogeneous, clutter edges and multiple interferers. Note that the clutter and interferer cells are set to be stable values in Figure 4. It only illustrates the relative position of the clutter edge and interferers.

4.1. Homogeneous Environment. Figure 5 shows the PD performance in a homogeneous environment for the VIE-CFAR along with results for an optimum detector (with a fixed detection threshold when the clutter power is known) and for the CA-CFAR, GO-CFAR, SO-CFAR, OS-CFAR $(k=21)$, and VI-CFAR. All of the CFAR detectors perform similarly and exhibit some CFAR loss relative to the optimum detector. The VIE-CFAR performs the same as VI-CFAR and behaves somewhat better than the SO- and OS-CFAR.

4.2. Variable Environment with Clutter Edge. In Figure 6, we evaluate the PFA performance of the proposed VIE-CFAR under the condition of clutter edges (as shown in Figures $4(\mathrm{~b})$ and $4(\mathrm{c}))$. Due to the adaptive selection procedure, both the VI-CFAR and VIE-CFAR behave steadily with the desired PFA. Particularly, when the number of clutter cells is more than $N / 2$ and the cell under test is from the clutter background, the PFA achieved by the VIE-CFAR and VICFAR is superior to the other CFAR processors.

4.3. Variable Environment with Multiple Interferers. In Figure 7, we assess the performance of the VIE-CFAR in the presence of a single interfering target that locates in one half of the reference window. The interferer is inserted in the 18th cell (as shown in Figure 4(d)). In this case, the performances of OS-CFAR, SO-CFAR, VI-CFAR, and VIE-CFAR are relatively unaffected while the performances of CA-CFAR and GO-CFAR degrade heavily. The degradation results from the fact that the interferer increases the estimated statistics and the decision thresholds of the CA-CFAR and GO-CFAR. We also observe that the VIE-CFAR and VI-CFAR perform nearly the same. This result is expected since they switch to the same reference window in this situation. The small CFAR loss in VI-CFAR and VIE-CFAR for low SNR is a result of errors in the window selection hypothesis. As the SNR increases, the VIE-CFAR selects the right reference cells and the performance approaches to the SO-CFAR and OS-CFAR.

In Figure 8, we present multiple interferers that locate in both halves of the reference window. In the case of two interfering targets (inserted in the 5th and 20th cells, as shown in Figure 4(e)), the VIE-CFAR and OS-CFAR retain good performances, while the other detectors show performance degradation. The performance degradation of VI-CFAR validates the necessity of introducing the excision procedure when the interfering targets are present in both halves of the reference window. When the SNR is low, the VIE-CFAR switches not always to the E-CFAR, and the performance is a bit lower than the OS-CFAR. As the SNR increases, the VIE-CFAR behaves similarly to the OSCFAR. As the number of interfering targets increases to 4 and locates in both halves of the reference window (the additional two interferers locate at the 7th and 18th cells, as shown in Figure $4(\mathrm{f})$ ), the performance of the OS-CFAR shows large degradation. The reason lies in that the value of $k$ in the OS-CFAR is not appropriate to tolerate enough interfering targets. The performance of VIE-CFAR maintains a stable level and is robust to the number of interferers.

Finally, we evaluate the PFA performance of the proposed VIE-CFAR in the presence of multiple interferers. From Figure 9(a), the proposed method controls the PFA within the same order of the designed PFA. This is because the VIE-CFAR scheme removes the multiple interferers by an excision procedure, and the resulting reference cells are under homogeneous environments. Figure 9(b) illustrates the variable index of the resulting reference cells. The variable index is lower than $K_{\mathrm{VI}}$, and accordingly the detector performs as CA-CFAR with the resulting homogeneous reference cells.

\section{Validation for Ship Detection on SAR Imagery}

Most of the operating ship detection systems, such as the search for unidentified maritime objects (SUMO) and ocean monitoring workstation (OMW), are developed from the CA-CFAR detector [3]. Therefore, we mainly conduct performance comparisons of the proposed method with the CACFAR and VI-CFAR detectors.

The proposed VIE-CFAR based ship detection method is validated on two frames of SAR imagery. The first one is acquired by ENVISAT on wide swath mode, centered at $37.83^{\circ} \mathrm{N}$ and $119.91^{\circ} \mathrm{E}$ (Yellow Sea, China) with a resolution of $12.5 \mathrm{~m} \times 12.5 \mathrm{~m}$ in the range and azimuth directions, respectively. The image size is $193 \times 228$ pixels and contains 39 visually inspected ships, as shown in Figure 10(a). The sea clutter environment presents clutter edges. The other one is acquired by TerraSAR-X over the area of Hong Kong, with a resolution of $1.9 \mathrm{~m} \times 3.3 \mathrm{~m}$ in the range and azimuth direction, respectively. The image size is $739 \times 887$ pixels and contains 41 visually inspected ships, as shown in Figure 11(a). The ships in this image are larger in pixels due to the higher resolution, and some of the ships are located closely. Both the ENVISAT and TerraSAR-X SAR images are level 2 products.

The test, guard, and background windows of the CFAR sliding stencil are set as 1 by 1,7 by 7 , and 13 by 13 for the ENVISAT image and 1 by 1,15 by 15 , and 21 by 21 for the TerraSAR-X image, respectively. The designed $P_{\mathrm{fa}}$ is set as 0.0001 . In the real SAR imagery, the INR and number of reference cells are larger than those in the simulations. Therefore, we set the $K_{\mathrm{VI}}$ and $K_{\mathrm{MR}}$ as 2.5 and 1.806 empirically. The excision factor $\lambda$ is determined by the iteration procedure.

For the ENVISAT image, detection results are illustrated in Figure 10. From Figures 10(b), 10(c), and 10(d), false alarms are observed for the CA-CFAR, VI-CFAR, and VIE-CFAR, and the number of false alarms is 3,2 , and 3, respectively. Note that the CFAR detection results are refined by morphological 


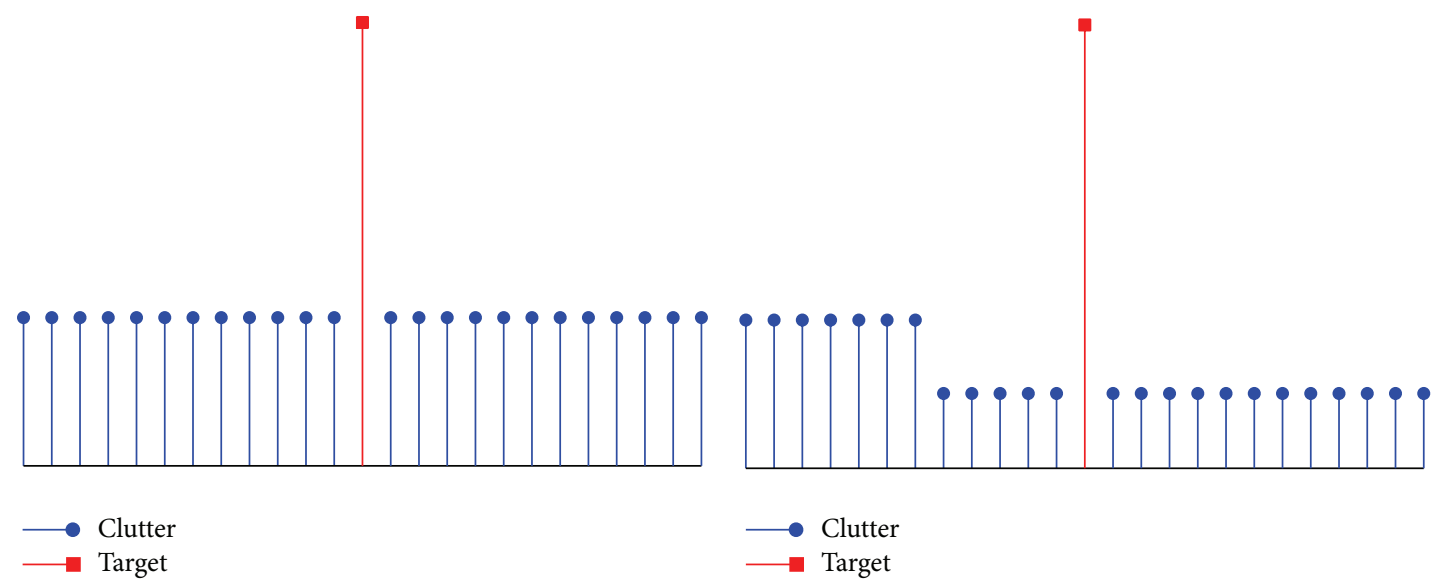

(a)

(b)

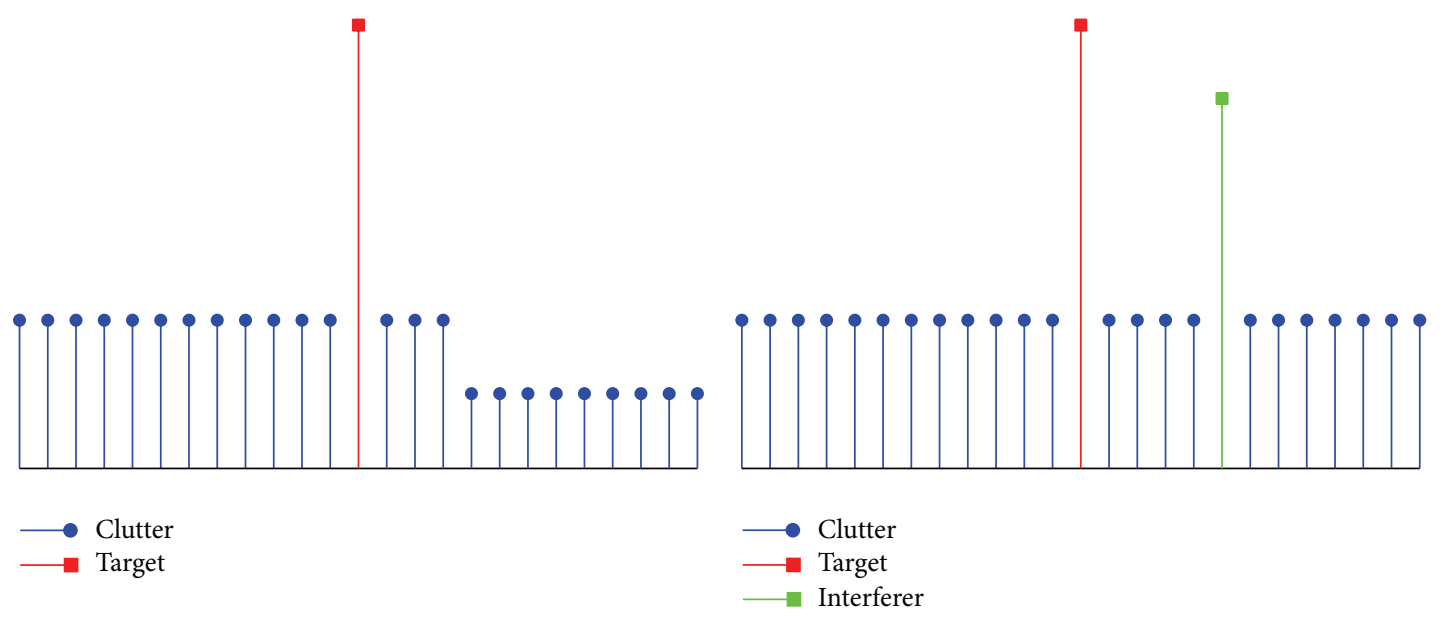

(c)

(d)

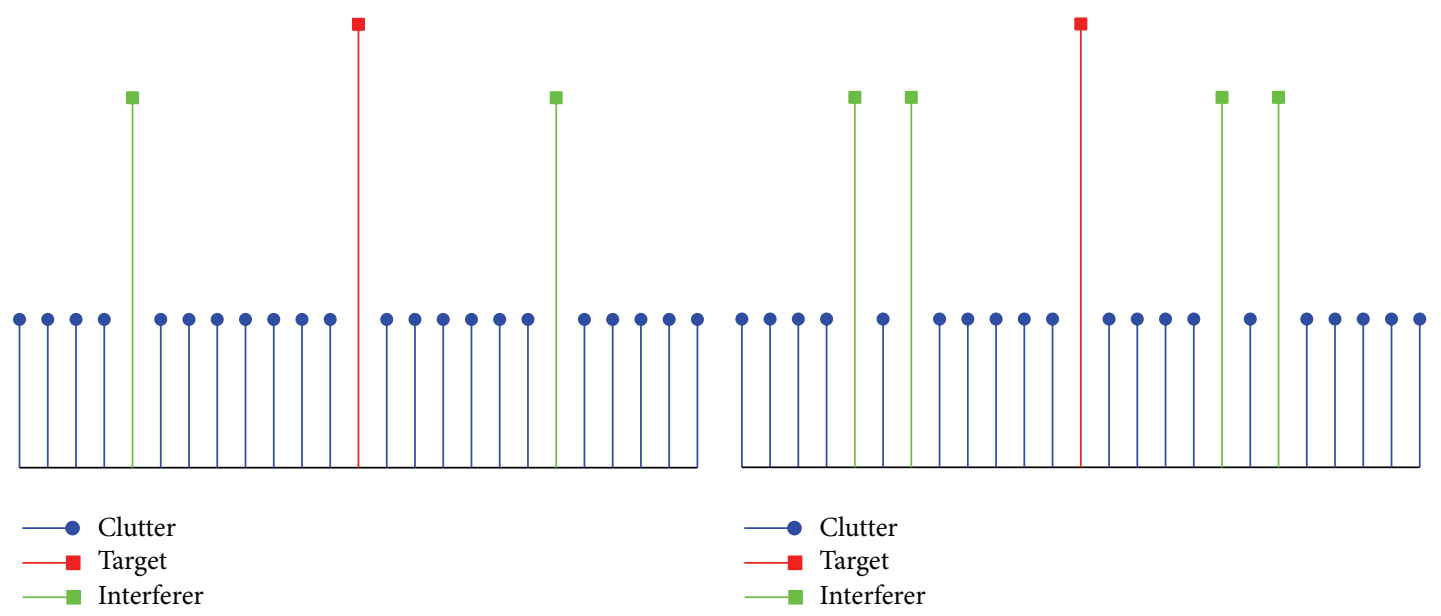

(e)

(f)

FIGURE 4: Illustration of the various simulated environments. (a) Homogeneous environment, (b) clutter edge on the leading window, (c) clutter edge on the lagging window, (d) one interferer on the lagging window (the 18th cell), (d) two interferers on both halves of the window (the 5th and 20th cells), and (f) four interferers on both halves of the window (the 5th, 7th, 18th, and 20th cells). 


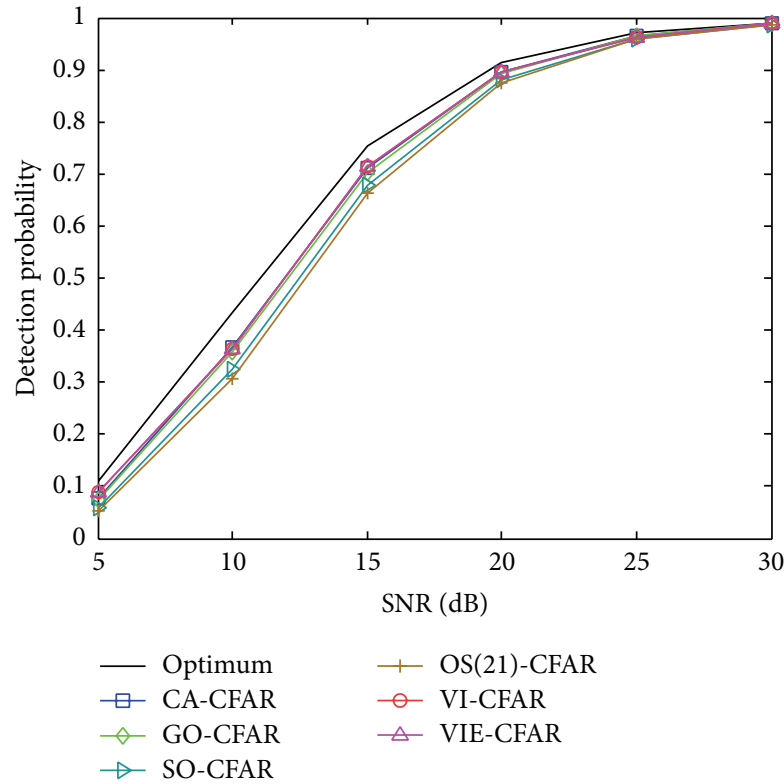

FIGURE 5: PD comparison of VIE-CFAR, optimum detector, CACFAR, GO-CFAR, SO-CFAR, OS-CFAR, and VI-CFAR in homogeneous environment.

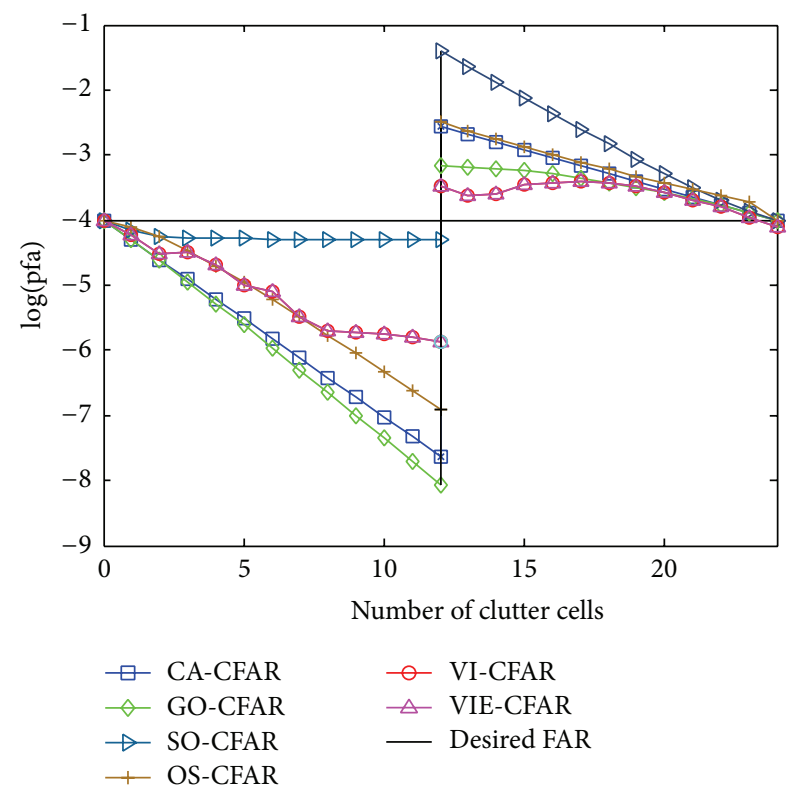

FIGURE 6: PFA comparison of VIE-CFAR, CA-CFAR, GO-CFAR, SO-CFAR, OS-CFAR, and VI-CFAR in the presence of clutter edges.

procedures. The number of false alarms produced by the proposed VIE-CFAR is consistent with the other two detectors in the final detection results.

In the TerraSAR-X SAR image, the closely located ships degrade the performances of CA-CFAR and VI-CFAR, as shown in Figures 11(b) and 11(c). Similar to the results of the ENVISAT image, the CFAR detection results of the TerraSAR-X image are also refined by morphological procedures. For the CA-CFAR, the ships number 6, 7, 8, 26, and 29 are misdetected. For the VI-CFAR, the ships number

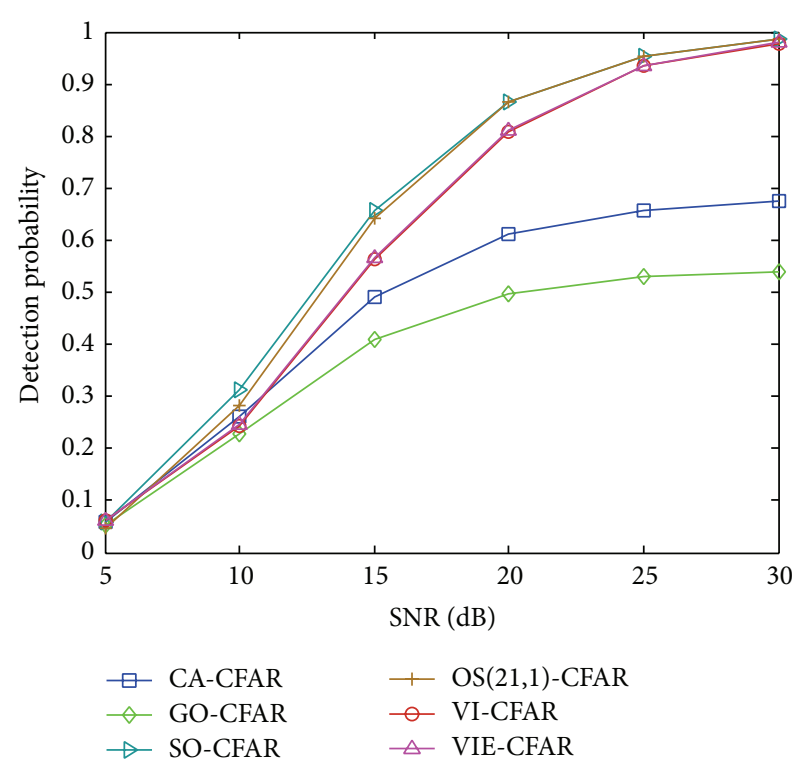

FIgURE 7: PD comparison of VIE-CFAR, CA-, GO-, SO-, OS-, and VI-CFAR for a single interfering target on one half of the reference windows.

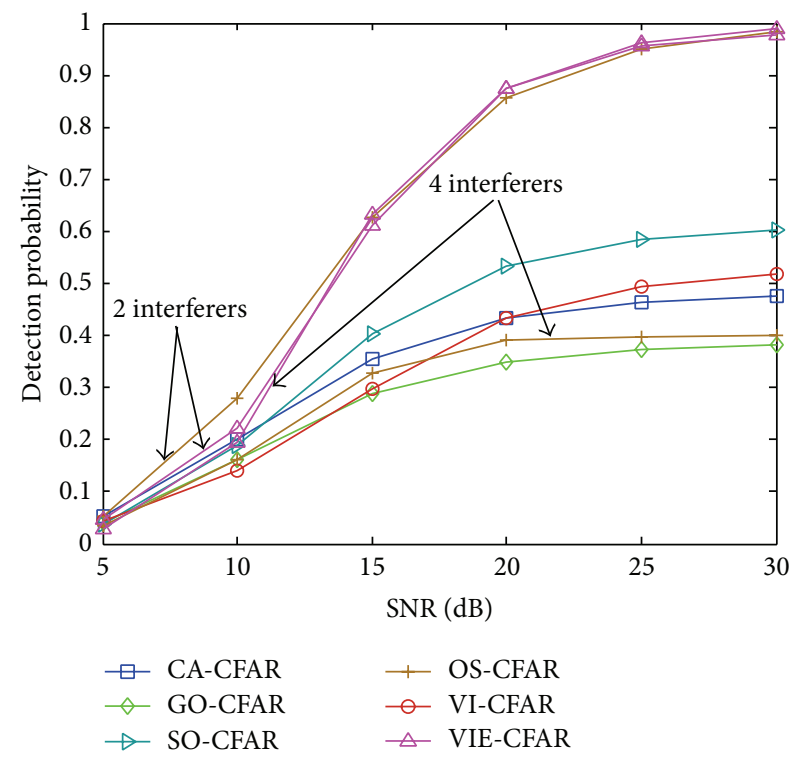

FIGURE 8: PD comparison of VIE-CFAR, CA-, GO-, SO-, OS-, and VI-CFAR for multiple interfering targets on both halves of the reference windows.

15,26 , and 29 are misdetected, and there are 6 additional false alarms. The VIE-CFAR also produces 5 false alarms; however, it alleviates the masking effect of the neighboring interferers and achieves highest PD among the detectors, as shown in Figure 11(d). In fact, the false alarms in the VICFAR and VIE-CFAR detection results are produced by high level intensity of sea clutters. These impulsive clutters cause false alarms intrinsically. Although the CA-CFAR produces fewer false alarms, there are 5 ships misdetected, which is unacceptable in practical marine surveillance applications. 

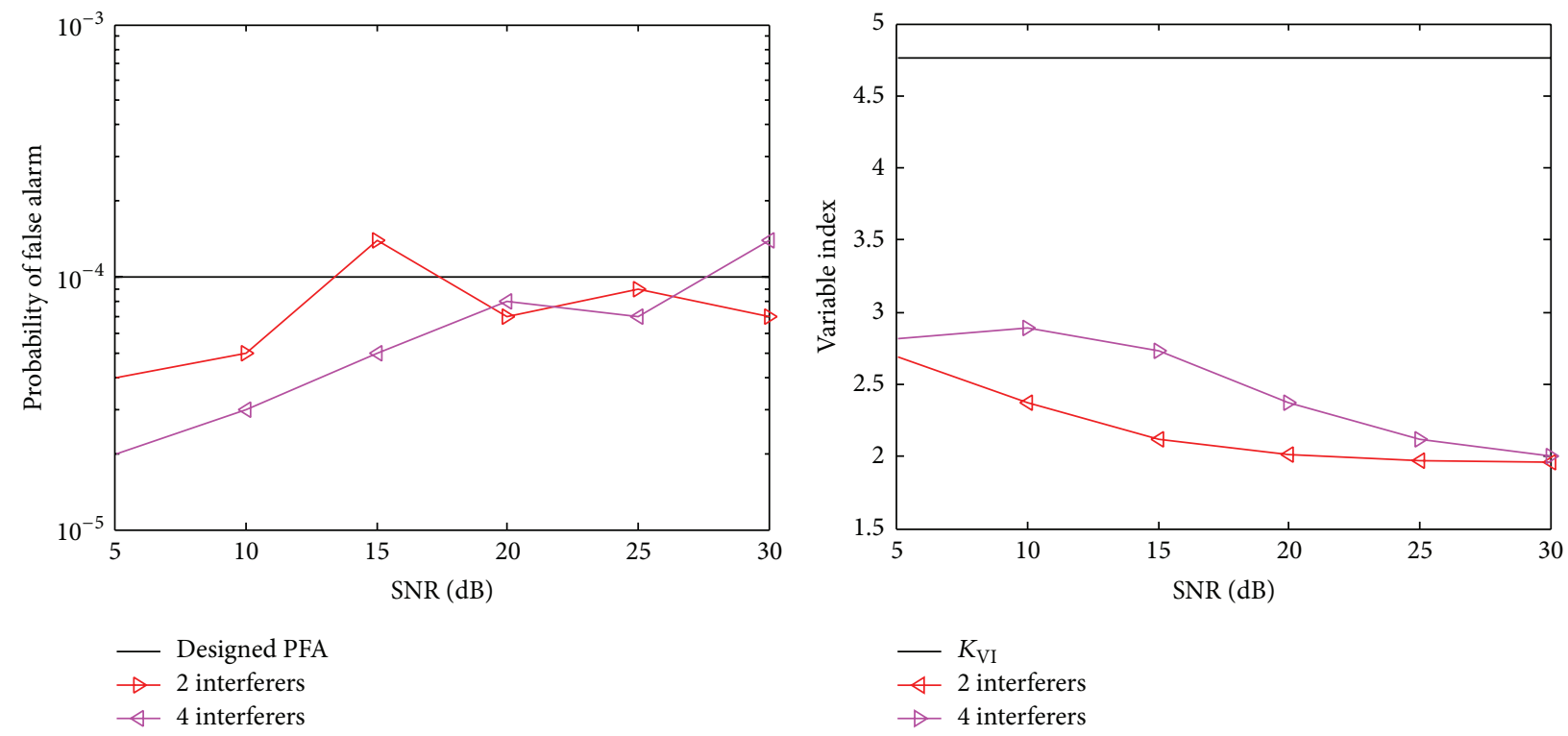

(a)

(b)

FIGURE 9: PFA performance of the proposed method with multiple interferers: (a) PFA and (b) threshold of the variable index.

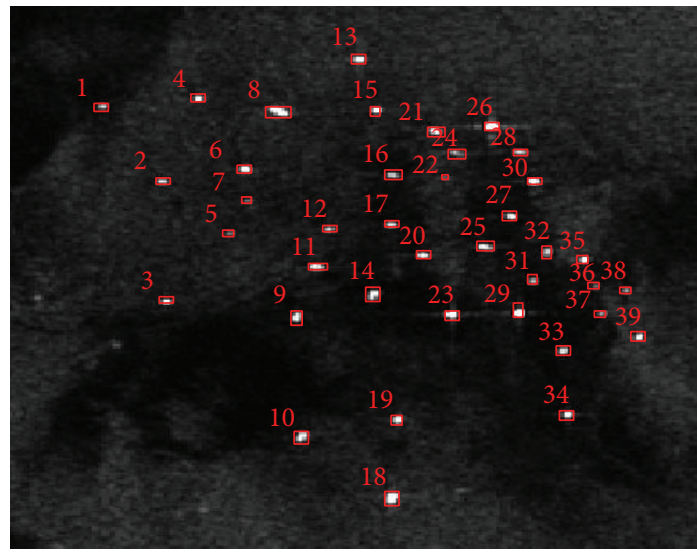

(a)

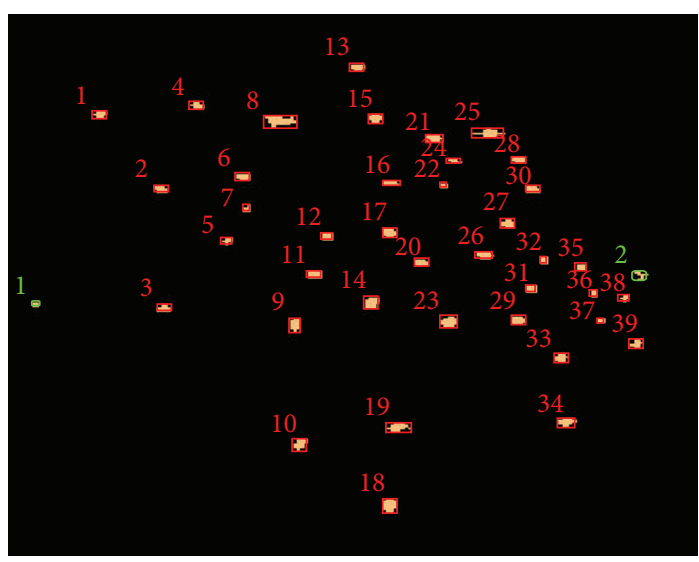

(c)

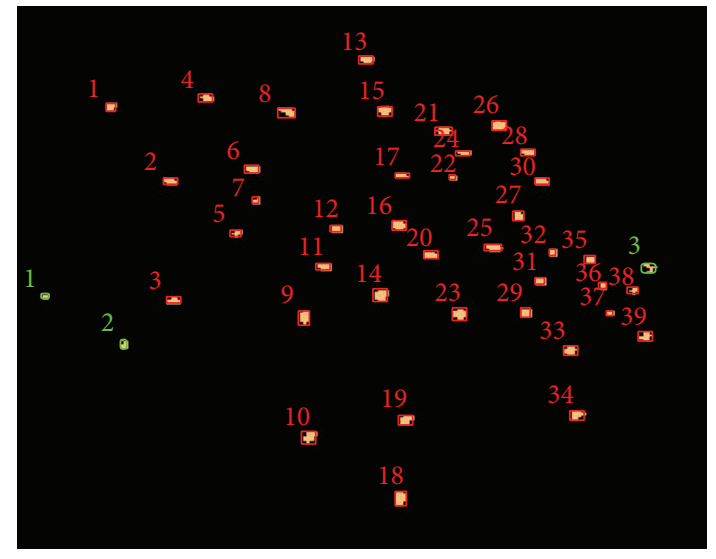

(b)

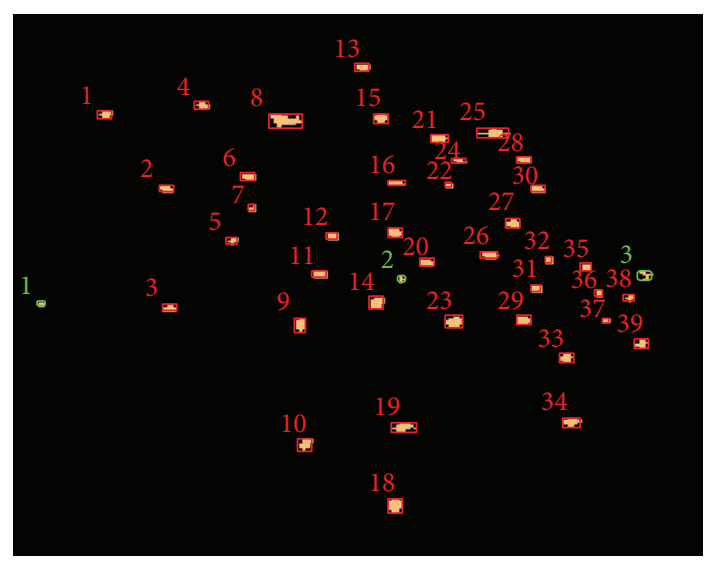

(d)

FIGURE 10: Detection results on ENVISAT image: (a) SAR image of the study area with visually inspected ships marked by red square, (b) CA-CFAR detection results, (c) VI-CFAR detection results, and (d) VIE-CFAR detection results. (red square: detected ships; green ellipse: false alarms; Cyan Ellipse: missing detection). 


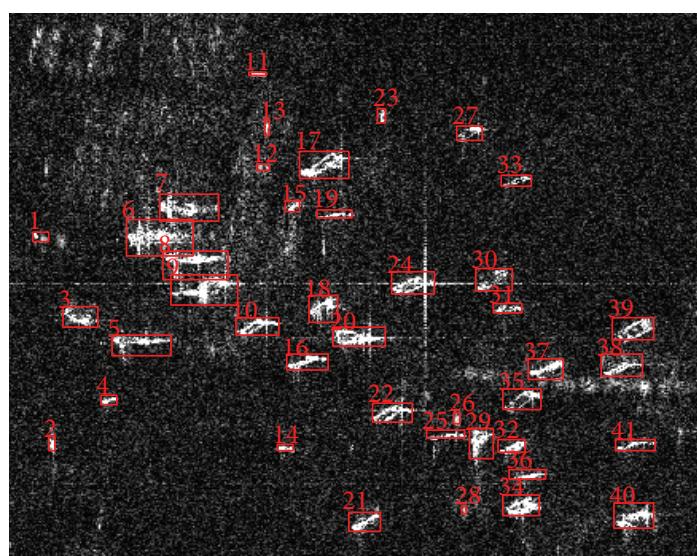

(a)

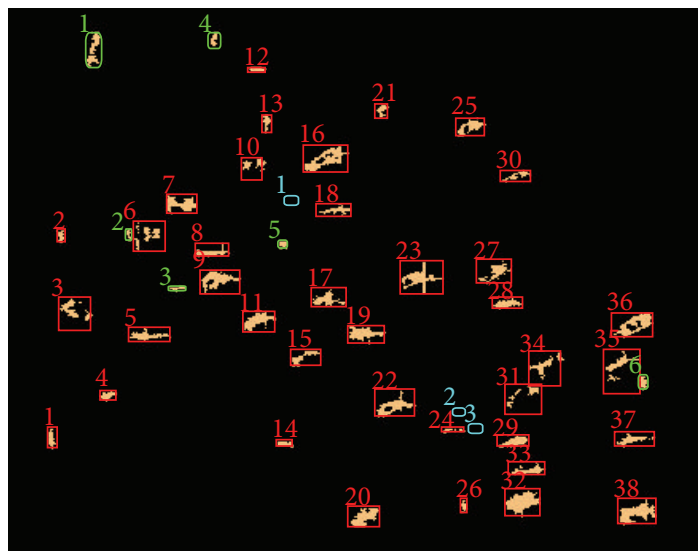

(c)

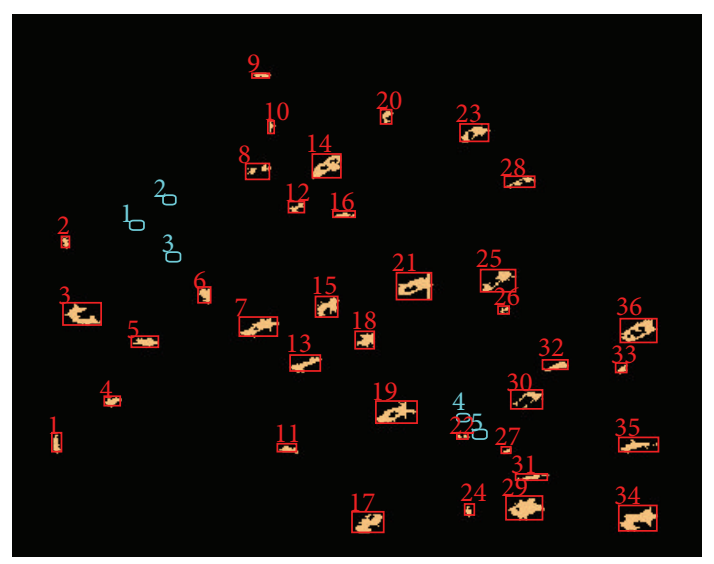

(b)

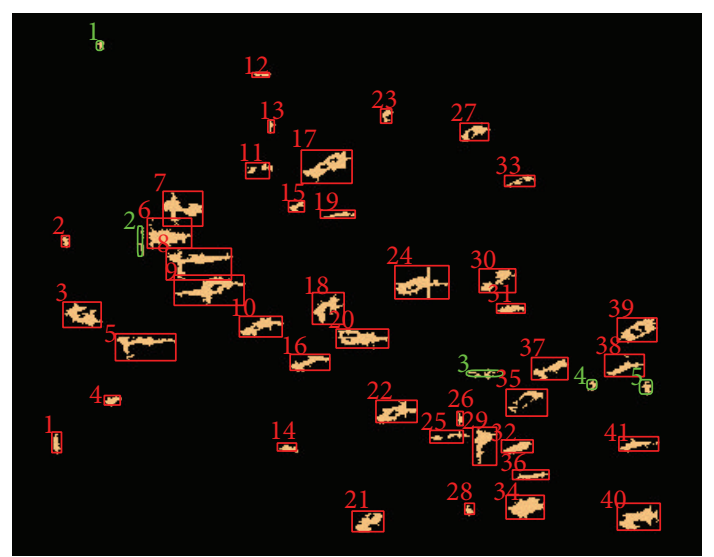

(d)

FIGURE 11: Detection results on TerraSAR-X SAR image with visually inspected ships marked by red square, (a) SAR image of the study area, (b) CA-CFAR detection results, (c)VI-CFAR detection results, and (d) VIE-CFAR detection results. (red square: detected ships; green ellipse: false alarms; Cyan Ellipse: missing detection).

TABLE 2: Overview of the detection results obtained by different detectors.

\begin{tabular}{lcccc}
\hline Detectors & Number of ships & Number of detected ships & Number of false alarms & Number of missed ships \\
\hline CA-CFAR & 80 & 75 & 3 & 5 \\
VI-CFAR & 80 & 77 & 8 & 3 \\
VIE-CFAR & 80 & 80 & 8 & 0 \\
\hline
\end{tabular}

Finally, we summarize the detection results on the ENVISAT and TerraSAR-X SAR imagery in Table 2. The results confirm that the VIE-CFAR is superior to VI-CFAR and CA-CFAR in terms of PD. The VIE-CFAR is more robust than the other detectors in the environment of multiple interferers.

\section{Conclusions}

In this paper, we have proposed a VIE-CFAR based ship detection method on SAR imagery. The VIE-CFAR improves the concept of VI-CFAR detection by introducing an excision procedure to alleviate the masking effect of multiple interferers. We have analyzed the performances of the VIECFAR detector under various environments and validated the proposed ship detection method on ENVISAT and TerraSAR-X SAR imagery. We conclude from the validation that the proposed VIE-CFAR is robust in the presence of multiple interferers compared to the VI-CFAR and CACFAR.

With the validation on real SAR imagery, the proposed VIE-CFAR behaves robustly in nonhomogeneous environments, especially in the presence of multiple interferers. Some additional remarks and discussions are given as follows.

(1) The VIE-CFAR accommodates the variety of environments and switches among several basic CFAR detectors dynamically. In paerticular, we introduce the excision procedure to address the multiple interferers that come from spiky sea clutters, ambiguities, and neighboring ships for ship detection on SAR imagery. Validation on ENVISAT and TerraSAR-X images illustrates the effectiveness of the proposed method. 
(2) The three-layer stencil is usually employed for ship detection on SAR imagery to avoid the ship pixels leaking to the clutter window. If the size of the stencil is set properly, it alleviates the masking effect of the ship itself. However, it is not able to account for the multiple interferers that we considered in this paper. The proposed VIE-CFAR based ship detection method alleviates the masking effect of the ship itself by employing the stencil and further addresses the challenges that come from multiple interferers by the excision procedure.

(3) Due to the window switching strategy and the additional excision procedure, the proposed VIE-CFAR consumes more computation than the CA-CFAR. However, the proposed method really produces better results in difficult scenarios. Once the method is embedded in operating ship detection system, fast algorithm and strategy can be designed to compensate the additional computation complexity.

(4) In the detection results, several kinds of false alarms such as spiky sea clutters, islands, and other manmade objects are facilitating to appear. They behave similarly in terms of image intensity with ships and are difficult to be removed by the CFAR method. In fact, polarimetric information has been introduced to analyze the different scattering mechanisms of ships and false alarms. However, it is out of the scope of this paper and can be investigated in future work.

(5) Ship surveillance with SAR images consists of several modules such as land masking, ship detection, parameter derivation, ship recognition, and cross validation with auxiliary data. We here focus on the detection stage and evaluate the performance of the proposed VIE-CFAR scheme. Future work will focus on embedding the proposed VIE-CFAR into a complete processing chain for ship surveillance with SAR imagery.

\section{Conflict of Interests}

The authors declare that there is no conflict of interests regarding the publication of this paper.

\section{Acknowledgment}

This work is partially supported by the National Natural Science Foundation of China under Grants 61372163 and 61240058.

\section{References}

[1] G. Margarit, J. J. Mallorqui, J. M. Rius, and J. Sanz-Marcos, "On the usage of GRECOSAR, an orbital polarimetric SAR simulator of complex targets, to vessel classification studies," IEEE Transactions on Geoscience and Remote Sensing, vol. 44, no. 12, pp. 3517-3525, 2006.

[2] A. Moreira, P. Prats-Iraola, M. Younis, G. Krieger, I. Hajnsek, and K. P. Papathanassiou, "A tutorial on synthetic aperture radar," IEEE Geoscience and Remote Sensing Magazine, vol. 1, no. 1, pp. 6-43, 2013.

[3] D. Crisp, "The State-of-the-Art in ship detection in synthetic aperture radar imagery," Tech. Rep. 013-053, Austrilian Defence Science and Technology Organisation, 2004.

[4] S. Brusch, S. Lehner, T. Fritz, M. Soccorsi, A. Soloviev, and B. Van Schie, "Ship surveillance with TerraSAR-X," IEEE Transactions on Geoscience and Remote Sensing, vol. 49, no. 3, pp. 10921103, 2011.

[5] J. Wei, P. Li, J. Yang, J. Zhang, and F. Lang, "A new automatic ship detection method using L-band polarimetric SAR imagery," IEEE Journal of Selected Topics in Applied Earth Observations and Remote Sensing, vol. 7, no. 4, pp. 1383-1393, 2014.

[6] A. Marino, "A notch filter for ship detection with polarimetric SAR data," IEEE Journal of Selected Topics in Applied Earth Observations and Remote Sensing, vol. 6, no. 3, pp. 1219-1232, 2013.

[7] G. Margarit, J. A. B. Milanés, and A. Tabasco, "Operational ship monitoring system based on Synthetic Aperture Radar processing," Remote Sensing, vol. 1, no. 3, pp. 375-392, 2009.

[8] M. Tello, C. Lopez-Martinez, J. Mallorqui, T. Tares, and H. Greidanus, "Advances in unsupervised ship detection with multiscale techniques," IEEE Geoscience and Remote Sensing Letters, vol. 2, no. 2, pp. IV-979-IV-982, 2009.

[9] S.-I. Hwang and K. Ouchi, "On a novel approach using MLCC and CFAR for the improvement of ship detection by synthetic aperture radar," IEEE Geoscience and Remote Sensing Letters, vol. 7, no. 2, pp. 391-395, 2010.

[10] H. M. Finn and R. S. Johnson, "Adaptive detection mode with threshold control as a function of spatially sampled clutter level estimates," RCA Review, vol. 29, pp. 414-468, 1968.

[11] P. P. Gandhi and S. A. Kassam, "Analysis of CFAR processors in homogeneous background," IEEE Transactions on Aerospace and Electronic Systems, vol. 24, no. 4, pp. 427-445, 1988.

[12] G. Gao, L. Liu, L. Zhao, G. Shi, and G. Kuang, "An adaptive and fast CFAR algorithm based on automatic censoring for target detection in high-resolution SAR images," IEEE Transactions on Geoscience and Remote Sensing, vol. 47, no. 6, pp. 1685-1697, 2009.

[13] R. Zhang, Y. Zou, W. Sheng, X. Ma, and H. Wang, "An improved CFAR detector for non-homogeneous clutter environment," in Proceedings of the International Symposium on Signals, Systems and Electronics (ISSSE '10), vol. 2, pp. 1-4, September 2010.

[14] M. E. Smith and P. K. Varshney, "Intelligent CFAR processor based on data variability," IEEE Transactions on Aerospace and Electronic Systems, vol. 36, no. 3, pp. 837-847, 2000.

[15] A. C. Frery, H.-J. Müller, C. D. C. F. Yanasse, and S. J. S. Sant'Anna, "A model for extremely heterogeneous clutter," IEEE Transactions on Geoscience and Remote Sensing, vol. 35, no. 3, pp. 648-659, 1997.

[16] H. Goldman and I. Bar-David, "Analysis and application of the excision CFAR detector," IEE Proceedings F Communications, Radar and Signal Processing, vol. 135, no. 6, pp. 563-575, 1988.

[17] G. Gao, "Statistical modeling of SAR images: a survey," Sensors, vol. 10, no. 1, pp. 775-795, 2010.

[18] R. Abdelfattah and J.-M. Nicolas, "Interferometric SAR coherence magnitude estimation using second kind statistics," IEEE Transactions on Geoscience and Remote Sensing, vol. 44, no. 7, pp. 1942-1953, 2006. 

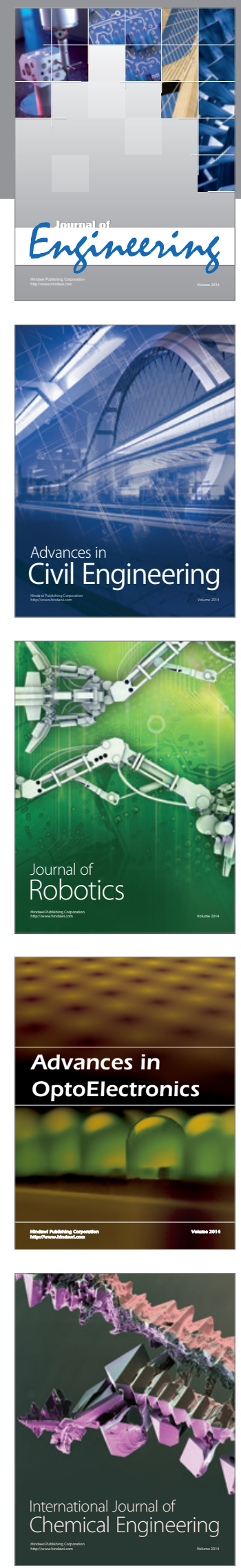

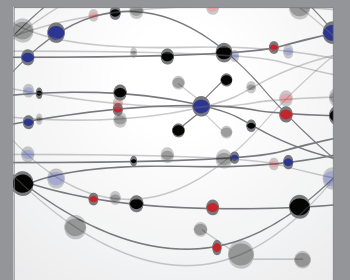

The Scientific World Journal
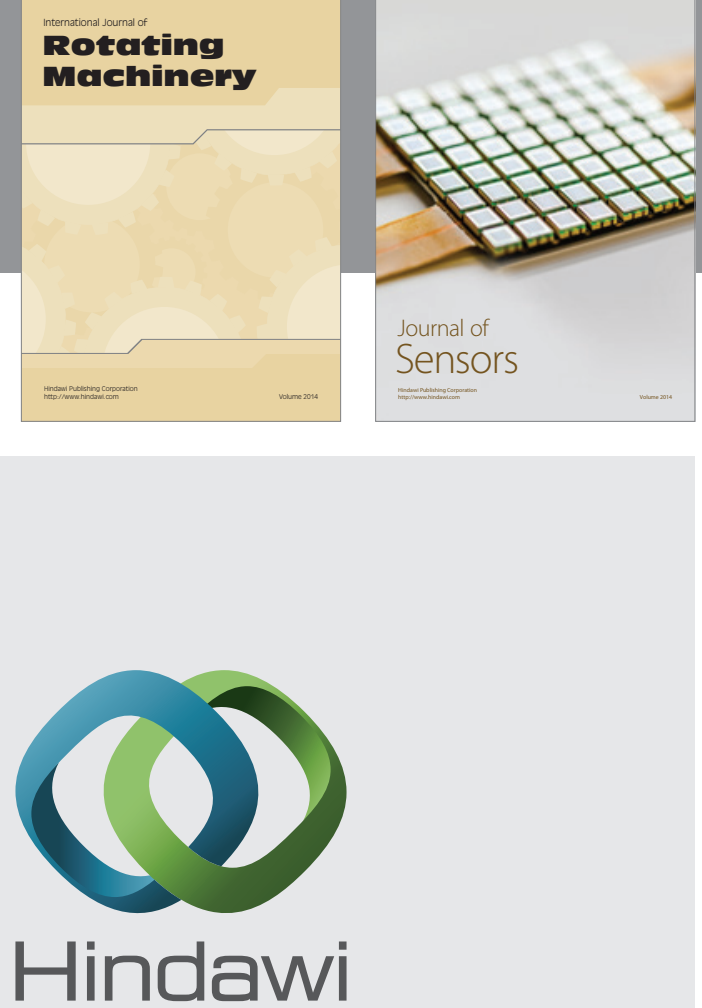

Submit your manuscripts at http://www.hindawi.com
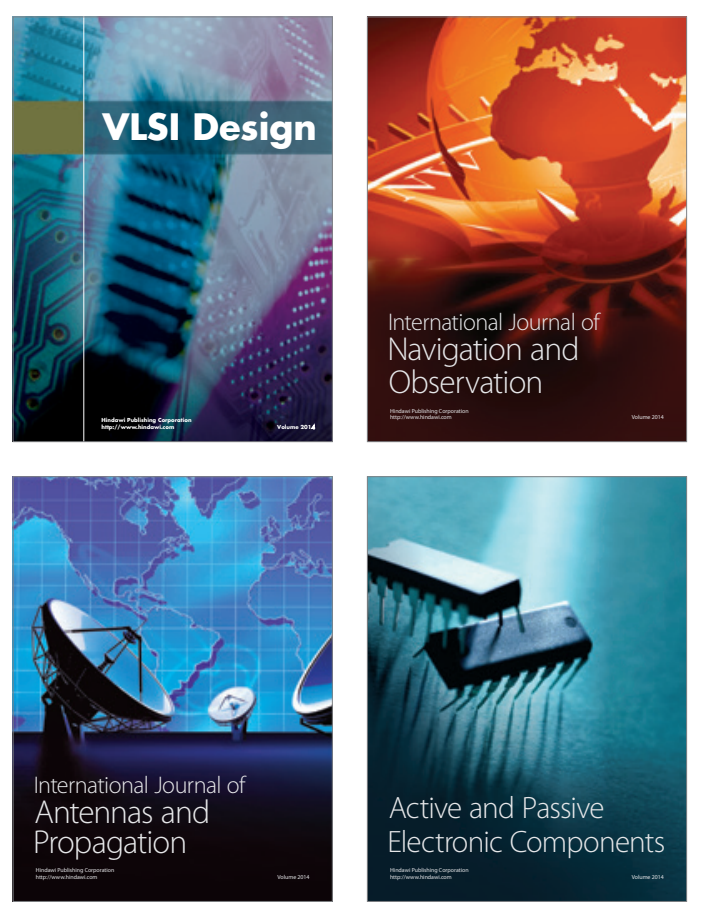
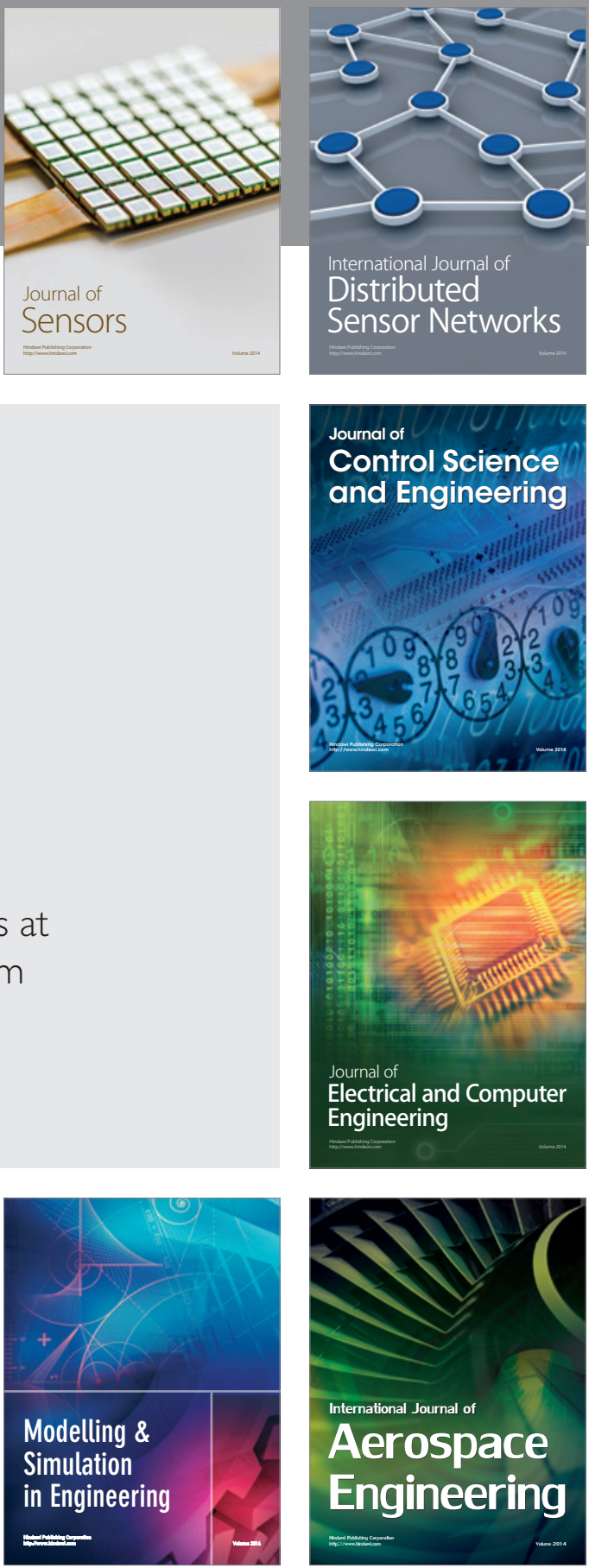

Journal of

Control Science

and Engineering
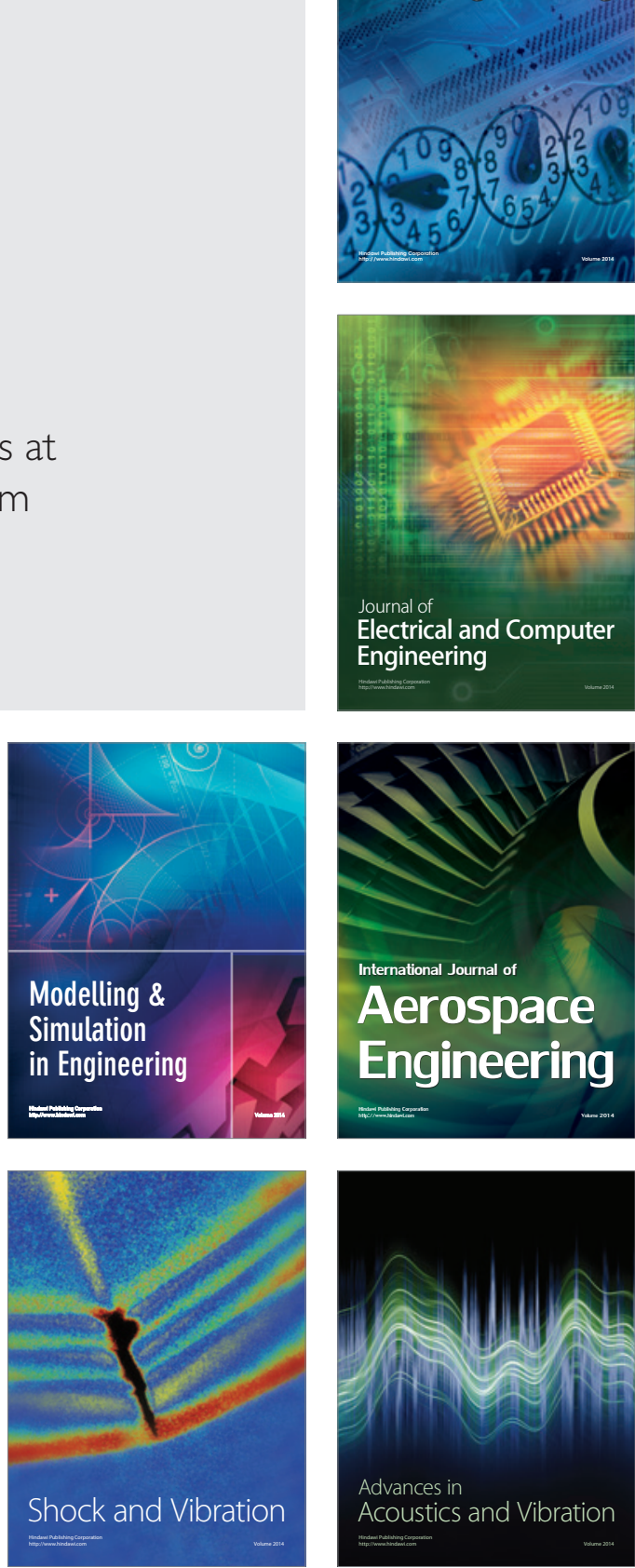